http://rev.med.panacea.unica.edu.pe

Rev méd panacea. 2013; 3(1): 6-10.

Recibido: 24 de Enero del 2013 | Aceptado: 27 de Marzo del 2013 | Publicado: 30 de Abril del 2013

CONFICTOS DE INTERÉS: NO DECLARADOS

ARTÍCULO ORIGINAL

\title{
Actitudes y barreras sobre investigación en atención primaria de salud de Ica
}

\author{
Bladimir Becerra-Canales ${ }^{1, a}$ \\ Área de Investigación en Atención Primaria de Salud, Red de Salud de Ica ${ }^{1}$ \\ Doctor en Salud Públicaa
}

O

bjetivo: Conocer las actitudes y barreras sobre investigación, percibidas por los profesionales en Atención Primaria de Salud (APS) de Ica. Materiales y métodos: Estudio descriptivo-relacional y de corte transversal, la muestra estuvo representada por 191 profesionales, que fueron seleccionados al azar, en 53 establecimientos del primer nivel de atención. Para establecer cuantitativamente el nivel de actitudes y percepción de barreras se aplicó una escala tipo Likert y un cuestionario respectivamente. Resultados: Se encontró actitud favorable en el $95.8 \%$ del total de la muestra; las mejores actitudes fueron: Para investigar no es necesario ser "un científico" (87.5\%), desean ampliar en el futuro sus conocimientos en MIC (91.7\%), darían tiempo fuera de su jornada laboral, para investigar (85.8\%), les interesa la investigación (89.5\%) etc. El $96.2 \%$ del total de los encuestados perciben en nivel alto, barreras de implementación de la investigación; los obstáculos identificados fueron la falta de tiempo (61.7\%), falta de motivación (86.3\%), no hay estructuras de apoyo (83.1\%), etc. Conclusiones: En APS de Ica, existen actitudes favorables hacia la investigación y se perciben niveles altos de barreras de implementación.

Palabras clave: Atención Primaria de Salud, investigación, actitudes, barreras (fuente: DeCS BIREME).

Autor corresponsal: Bladimir Becerra Canales. Dirección: CC.HH La Angostura III Etapa H-10, Ica Perú. Teléfono: (51) 956690060. Correo electrónico: icapredica@gmail.com

\section{INTRODUCCIÓN}

En la región Ica, una pobre formación en funciones del equipo de atención primaria, investigación recibida en pre grado y la lo cierto es que aún no está implantada ni ausencia de solidas líneas de investigación asumida; con la reforma de la atención pridan como resultado el escaso número de maria (1981) se pusieron las bases que investigaciones realizadas aun en el post podían propiciar la investigación, sin embargrado, esto sumado a la falta de capaci- go la presencia de la investigación en la APS tación en temas de redacción científica y la y de la APS en la investigación es débil; más migración de profesionales que sirvan como aún si tenemos en cuenta, que en nuestra modelos formativos en publicación, se con- región, en este ámbito asistencial, labora vierte en un problema que debe ser afron- una gran proporción de los profesionales del tado por las autoridades sanitarias y/o sector salud, un colectivo joven abierto a las académicas (1).

La producción científica biomédica de la con estudios de Maestría y Doctorado, el 70 región, de trece años revisados (1998-2010) - 80\% de los motivos de consulta de la es fácilmente superada por publicaciones población se atiende en el primer nivel de realizadas por estudiantes de medicina de atención, en donde se genera además el Chile, en revistas médicas indezadas a Sci- consumo de más del 60\% de los medicamenELO, que llegan de 50 a 70 por año, y repre- tos(3).

senta en promedio solo el $8 \%$ de la produc- Se ha observado en algunos integrantes del ción científica realizada por docentes de la equipo multidisciplinario de APS de Ica, comFacultad de Medicina de la Universidad portamientos y manifestaciones verbales de Nacional Mayor de San Marcos, durante diez aceptación, rechazo o indiferencia hacia la años (2).

Aunque el marco legal que sustenta la reforma de la Atención Primaria en Salud (APS) en el Perú, incluye la investigación entre las
En tanto que no tenemos datos referentes a las actitudes y barreras sobre Investigación en APS de Ica, se desarrolló el estudio con el objetivo de Conocer las actitudes y barreras sobre investigación, percibidas por los profesionales en APS de Ica.

\section{MATERIALES Y MÉTODOS}

Se realizó un estudio descriptivo correlacional de corte transversal, la muestra estuvo representada por 191 profesionales (59 Médicos, 38 Odontólogos, 26 Obstetras, 42 Enfermeros y 26 Químicos Farmacéuticos), de una población de 252 profesionales del primer nivel de atención de la Red de Salud de Ica.

El procedimiento empleado para la selección de la muestra fue el probabilístico y las unidades de la muestra fueron elegidas al azar.

Se aplicó un instrumento para obtener información concerniente a: Sección I. Información general personal ( 05 ítems).

Sección II. Actitudes con respecto a la investigación (15 ítems) los cuales fueron calificados con puntuación de Likert de 1 a 5; y la puntuación total fluctúa entre 15 y 75 puntos, considerándose actitud favorable $\geq$ 45 puntos. Sección III. Percepción de barreras de implementación de la investigación (10 ítems), considerándose Nivel de barrera alto, si responden afirmativamente $\leq 4$ preguntas realizadas.

La sección que corresponde a la escala tipo Likert (sección II), se construyó generando un elevado número de afirmaciones que calificaron al objeto de actitud (40 Ítems) y se administró a un grupo de trabajadores (28), del Puesto de Salud Fonavi IV, para obtener las puntuaciones del grupo en cada afirmación. Estas puntuaciones se correlacionaron con las puntuaciones del grupo a toda la escala (la suma de las puntuaciones de todas las afirmaciones), y las afirmacio- 
afirmaciones cuyas puntuaciones se correlacionaron significativamente con las puntuaciones de toda la escala, se seleccionó para integrar el instrumento de medición.

Las etapas para la elaboración de las preguntas (sección III), fueron las recomendadas por Sampieri 1997 (p-236): Decisión sobre la información a investigar, decisión sobre el tipo de cuestionario que mejor se adaptara a la información a recoger, redacción de un primer borrador, examen del borrador y revisión de las preguntas.

Respecto al tipo de preguntas, abiertas o cerradas, decidimos inclinarnos por el tipo de pregunta cerrada, ya que requiere menor tiempo para su contestación y genera una más rápida codificación.

Se establecieron mecanismos de control de calidad y confiabilidad de la información a través de la validación del instrumento por jueces o de Delfos, a quienes se les proporcionó una Lista de Cotejo, para que evalúen los ítems uno por uno en términos de: Relevancia, coherencia, suficiencia y claridad. Y el Índice de Consistencia Interna de Alfa de Cronbach (Método de la Varianza de los Ítem. $a=0.802$ ).

El área de estudio fue 53 establecimientos del Primer Nivel de Atención de la Red de Salud de Ica. Los profesionales participantes en el estudio, llenaron los formularios de encuesta, el mismo que fue auto aplicado, en el mes de Agosto del 2012. Se tomó en cuenta aspectos éticos, como el permiso a las jefaturas de los centros y puestos de salud, donde laboran los profesionales y se indicó que su participación es voluntaria, anónima y en consecuencia sus respuestas no serán divulgadas en forma individual sino colectiva en gráficos o tablas. Asimismo que el personal podrá dejar de contestar aquellas preguntas que no desee.

La base de datos fue sometido a un análisis estadístico, utilizando el paquete estadístico Statistical Package for the Social Sciences (SPSS) para Windows versión 17.0 en Español. Se calcularon las distribuciones de frecuencias por variables y se realizó un análisis univariado y bivariado, se estimó la normalidad de la distribución de las variables mediante la prueba de Kolmogorov-Smirnov y se realizó un análisis no paramétrico, con el estadístico de prueba de U de Mann-Whitney.

\section{RESULTADOS}

En general la actitud fue favorable en el $95.8 \%$ (183/191) del total de la población de estudio, distribuidos por grupos ocupacionales se tienen: El 96.6\%(57/59) de Médicos; $94.5 \%$ (36/38) de Odontólogos, 97.6\%(41/42) de Enfermeros, $92.3 \%(24 / 26)$ de Obstetras y $96.2 \%$ (25/26) de Químicos farmacéuticos.
Tabla 1. Nivel de actitud, según grupo ocupacional

\begin{tabular}{lcc}
\multirow{2}{*}{ Actitud } & Desfavorable & Favorable \\
\cline { 2 - 3 } & $\mathrm{N}(\%)$ & $\mathrm{N}(\%)$ \\
\hline Médico & $2(3,4)$ & $57(96,6)$ \\
Odontólogo & $2(5,3)$ & $36(94,7)$ \\
Enfermero & $1(2,4)$ & $41(97,6)$ \\
Obstetra & $2(7,7)$ & $24(92,3)$ \\
Químico F. & $1(3,8)$ & $25(96,2)$ \\
\hline
\end{tabular}

Según el estadístico de prueba de U de Mann -Whitney, no se aprecia diferencias significativas de las actitudes según grupo ocupacional de profesionales ( $p$-valor $=0.732)$. Así mismo según sexo $(p$-valor $=0.705)$, grado académico ( $p$-valor $=0.133)$, edad $(p$-valor $=$ Tabla 2. Resultados generales de las actitudes hacia la investigación

\begin{tabular}{|c|c|c|c|c|c|}
\hline Item & $\begin{array}{c}\text { Totalmen- } \\
\text { te en } \\
\text { desacuer- } \\
\text { do }\end{array}$ & $\begin{array}{l}\text { En } \\
\text { desacuer- } \\
\text { do }\end{array}$ & $\begin{array}{c}\text { Ni de } \\
\text { acuerdo } \\
\text { ni en } \\
\text { desacuer- } \\
\text { do }\end{array}$ & $\begin{array}{l}\text { De } \\
\text { acuer- } \\
\text { do }\end{array}$ & $\begin{array}{l}\text { Total- } \\
\text { mente de } \\
\text { acuerdo }\end{array}$ \\
\hline $\begin{array}{l}\text { 1. Para investigar es necesario ser "un } \\
\text { científico" una persona que tiene un nivel } \\
\text { académico bastante alto. }\end{array}$ & $35.1 \%$ & $52.4 \%$ & $5.8 \%$ & $6.3 \%$ & $0.5 \%$ \\
\hline $\begin{array}{l}\text { 2. Deseo ampliar en el futuro mis conoci- } \\
\text { mientos en Metodología de la Investi- } \\
\text { gación científica. }\end{array}$ & $1.0 \%$ & $2.1 \%$ & $5.2 \%$ & $59.2 \%$ & $32.5 \%$ \\
\hline $\begin{array}{l}\text { 3. Daria tiempo fuera del horario de tra- } \\
\text { bajo para investigar }\end{array}$ & $1.0 \%$ & $5.8 \%$ & $7.3 \%$ & $65.4 \%$ & $20.4 \%$ \\
\hline $\begin{array}{l}\text { 4. Es posible realizar investigación de } \\
\text { calidad en los Centros y Puestos de salud }\end{array}$ & $1.0 \%$ & $5.8 \%$ & $7.9 \%$ & $52.4 \%$ & $33.0 \%$ \\
\hline $\begin{array}{l}\text { 5. Pienso implicarme en el futuro en un } \\
\text { trabajo de investigación. }\end{array}$ & $0.5 \%$ & $4.2 \%$ & $14.7 \%$ & $65.4 \%$ & $15.2 \%$ \\
\hline $\begin{array}{l}\text { 6. Es necesario formar en investigación a } \\
\text { los profesionales que van a investigar }\end{array}$ & $0.0 \%$ & $6.8 \%$ & $4.7 \%$ & $59.2 \%$ & $29 \cdot 3 \%$ \\
\hline $\begin{array}{l}\text { 7. Considero que en forma paralela la } \\
\text { investigación debe ser una actividad más } \\
\text { del trabajo asistencial. }\end{array}$ & $1.0 \%$ & $18.8 \%$ & $10.5 \%$ & $57.6 \%$ & $12.0 \%$ \\
\hline 8. Me agrada la actividad de investigar & $0.5 \%$ & $3.1 \%$ & $10.5 \%$ & $67.0 \%$ & $18.8 \%$ \\
\hline $\begin{array}{l}\text { 9. Se aprende mucho con la investigación } \\
\text { científica }\end{array}$ & $1.6 \%$ & $2.6 \%$ & $3.1 \%$ & $51.3 \%$ & $41.4 \%$ \\
\hline $\begin{array}{l}\text { 10. Podría ser un profesional más compe- } \\
\text { tente si aprendo a investigar }\end{array}$ & $1.6 \%$ & $7 \cdot 3 \%$ & $10.5 \%$ & $44 \cdot 5 \%$ & $36.1 \%$ \\
\hline $\begin{array}{l}\text { 11. Me considero capacitado para comen- } \\
\text { zar un trabajo de investigación. }\end{array}$ & $1.0 \%$ & $17.3 \%$ & $22.0 \%$ & $47.1 \%$ & $12.6 \%$ \\
\hline $\begin{array}{l}\text { 12. Siento que tengo las habilidades para } \\
\text { ser investigador }\end{array}$ & $1.0 \%$ & $3.1 \%$ & $18.8 \%$ & $67.0 \%$ & $9.9 \%$ \\
\hline $\begin{array}{l}\text { 13. La investigación tiene que ver con mi } \\
\text { carrera profesional }\end{array}$ & $1.6 \%$ & $4.2 \%$ & $7.9 \%$ & $59.2 \%$ & $27.2 \%$ \\
\hline $\begin{array}{l}\text { 14. La investigación es una pérdida de } \\
\text { tiempo }\end{array}$ & $56.0 \%$ & $39 \cdot 3 \%$ & $4.2 \%$ & $0.5 \%$ & $0.0 \%$ \\
\hline 15. No me interesa la investigación. & $41.9 \%$ & $47.6 \%$ & $7.3 \%$ & $2.6 \%$ & $0.5 \%$ \\
\hline
\end{tabular}

0.862) y condición laboral ( $p$-valor = 0.934). las barreras para el desarrollo de investigación, se realizó inicialmente excluyendo a aquellos sujetos que habían reportado no estar interesados en realizar investigación, los mismos que fueron el $4.2 \%(8 / 191)$ del total de los encuestados.

Se constata que el 96.2\%(176/183) del total de encuestados, en un porcentaje considerable, perciben en un nivel alto, barreras para el desarrollo de la investigación; así mismo el 100\%(57/57) de Médicos; el 97.2\%(35/36) de Odontólogos, el 87.8\%(36/41) de Enfermeros, el $95.8 \%(23 / 24)$ de Obstetras y el 100\%(25/25) de Químicos farmacéuticos.

Según la prueba estadística de U de MannWhitney, no se aprecia diferencias significatiEl análisis de la sección dedicada a identificar 


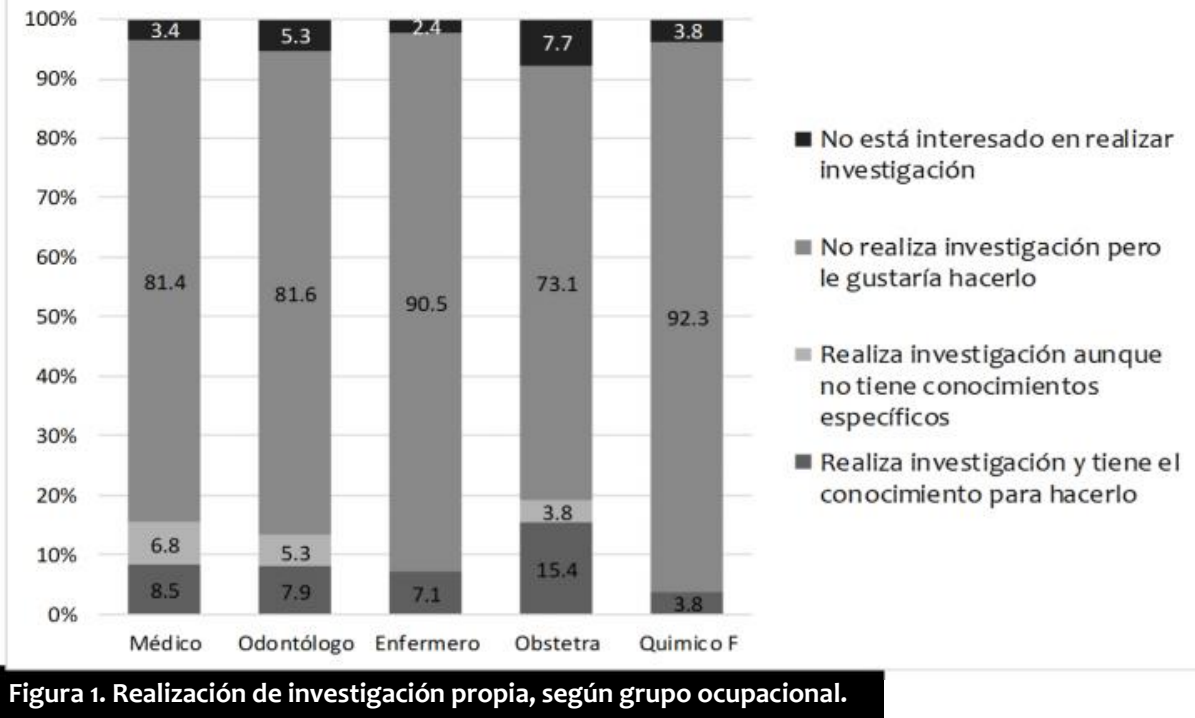

Tabla 3. Nivel de barrera, según grupo ocupacio-

\begin{tabular}{lcc} 
& N. Alto & N. Bajo \\
\cline { 2 - 3 } & $\mathrm{N}(\%)$ & $\mathrm{N}(\% / 9$ \\
\hline Médico & $57(100)$ & $0(0)$ \\
Odontólogo & $35(97,2)$ & $1(2,8)$ \\
Enfermero & $36(87,8)$ & $5(12,2)$ \\
Obstetra & $23(95,8)$ & $1(4,2)$ \\
Químico F. & $25(100)$ & $0(0)$ \\
\hline
\end{tabular}

tigación a los profesionales que van a investigar $(88.5 \%$, resultados que se corresponde con un estudio realizado en el Perú, donde se concluye que las estrategia para incrementar el número de investigaciones, debieran enfocarse en el fortalecimiento de las bases teóri-

ocupacional de profesionales ( $\mathrm{p}$-valor $=$ 0.732). Así mismo según sexo ( $p$-valor $=$ $0.705)$, grado académico ( $p$-valor $=0.133)$, edad $(p$-valor $=0.862)$ y condición laboral $(p$ valor $=0.934)$.

Tabla 4. Resultados generales de la percepción de barreras, para el desarrollo de la investigación.

El análisis de la sección dedicada a identificar las barreras para el desarrollo de investigación, se realizó inicialmente excluyendo a aquellos sujetos que habían reportado no estar interesados en realizar investigación, los mismos que fueron el $4.2 \%(8 / 191)$ del total de los encuestados.

Se constata que el $96.2 \%(176 / 183)$ del total de encuestados, en un porcentaje considerable, perciben en un nivel alto, barreras para el desarrollo de la investigación; así mismo el 100\%(57/57) de Médicos; el 97.2\%(35/36) de Odontólogos, el 87.8\%(36/41) de Enfermeros, el $95.8 \%(23 / 24)$ de Obstetras y el $100 \%(25 / 25)$ de Químicos farmacéuticos.

Según la prueba estadística de $U$ de MannWhitney, no se aprecia diferencias significativas del nivel barreras según grupo ocupacional de profesionales ( $\mathrm{p}$-valor $=0.268$ ); así mismo según sexo $(p$-valor $=0.327)$, grado académico ( $p$-valor $=0.150)$, según edad se aprecia diferencias significativas de la percepción de barreras hacia la investigación ( $p$ valor $=0.007)$; así mismo según condición laboral $(p$-valor $=0.048)$.

\section{DISCUSIÓN}

El principal fin de la investigación es mejorar la práctica asistencial aplicando métodos científicos para la resolución de las preguntas y dudas relevantes que surgen cotidianamente en los Centros de Salud (4). Hasta ahora, disponíamos de escasos datos acerca de cuál es la actitud hacia la investigación y la percepción sobre las barreras que impiden investigar a los profesionales de APS de Ica. Conociéndolas podremos estar en disposición de diseñar estrategias que aumenten la disposi-

\begin{tabular}{|c|c|c|}
\hline Item & No & Si \\
\hline $\begin{array}{l}\text { 1. Tiene el tiempo necesario dentro de su trabajo asistencial, para realizar proyectos } \\
\text { de investigación científica. }\end{array}$ & $617 \%$ & $38.3 \%$ \\
\hline 2. Ha asistido alguna vez a un curso sobre M etodología de la Investigación Científica. & $42.6 \%$ & $57.4 \%$ \\
\hline $\begin{array}{l}\text { 3. En su establecimiento de salud, se programan actividades de investigación científi- } \\
\text { ca en el PpR, POI, PSL, CONTRATOS etc. }\end{array}$ & $100.0 \%$ & $0.0 \%$ \\
\hline $\begin{array}{l}\text { 4. Si realiza un trabajo de investigación, cree usted que será reconocido o valorado } \\
\text { adecuadamente por los jefes. }\end{array}$ & $54.1 \%$ & $45.9 \%$ \\
\hline $\begin{array}{l}\text { 5. Los jefes inmediatos motivan al personal, para hacer trabajos de investigación } \\
\text { científica. }\end{array}$ & $86.3 \%$ & $13.7 \%$ \\
\hline $\begin{array}{l}\text { 6. La Red de Salud de Ica, cuenta con estructuras de apoyo a la investigación en } \\
\text { Atención Primaria (Oficinas, áreas o unidades de investigación científica) }\end{array}$ & $83.1 \%$ & $16.9 \%$ \\
\hline $\begin{array}{l}\text { 7. Existen tutores y/o asesores de investigación en la Red de Salud de Ica, para facili- } \\
\text { tar trabajos de investigación. }\end{array}$ & $82.5 \%$ & $17.5 \%$ \\
\hline $\begin{array}{l}\text { 8. Los establecimientos de salud cuentan con recursos económicos para el financia- } \\
\text { miento de trabajos de investigación científica }\end{array}$ & $96.7 \%$ & $3.3 \%$ \\
\hline $\begin{array}{l}\text { 9. Los profesionales de los Centros y Puestos de salud, actualmente realizan proyec- } \\
\text { tos de investigación, como parte de su trabajo asistencial }\end{array}$ & $99.5 \%$ & $0.5 \%$ \\
\hline $\begin{array}{l}\text { 10. Conoce y/o pertenece a algún grupo de investigación en Atención Primaria de } \\
\text { Salud }\end{array}$ & $92.9 \%$ & $7.1 \%$ \\
\hline
\end{tabular}

ción y motivación de los mismos a incorporar la investigación en nuestro ámbito profesional.

En general la actitud fue favorable (95.8\%), hacia la investigación $(5,6,7,8)$. Las mejores actitudes encontradas fueron: Para investigar no es necesario ser "un científico" (87.5\%), datos que no se corresponde con los hallados en Guatemala; donde la población objeto de estudio imaginan al investigador como "un científico,...una persona superprofesional que tiene un nivel académico bastante alto."(3).

Los profesionales de APS de Ica, desean amdología de la Investigación Científica (MIC) tes en el estudio expresaron un alto nivel de $(917 \%$; consideran necesario formar en inves- interés $(89.5 \%$ y agrado $(85.8 \%$, hacia la in- 
interés $(89.5 \%$ y agrado $(85.8 \%$, hacia la investigación (11, 12).

Como en la mayor parte de los estudios publicados tanto en España como en otros países $(13,11,7,14,15,16)$, la escasez de tiempo $(617 \%$ p para dedicar a la investigación se constituye en el principal obstáculo que los profesionales de APS de Ica, reconocen para desarrollarla. Aunque algunos autores distinguen entre falta de tiempo y presión asistencial excesiva, entendemos que el problema realmente se concreta en que el profesional, hoy por hoy, necesita dedicar su tiempo personal para realizar tareas de investigación, siendo prácticamente imposible realizarlas en el tiempo laboral(6).

Aunque a gran distancia del factor tiempo y coincidiendo con lo apuntado por otros autores $(14,17)$ la falta de motivación $(86.3 \%$, incluyendo aquí la falta de reconocimiento profesional $(54.1 \%$, se constituye también en un obstáculo importante para investigar. A este respecto, creemos que la valoración de la actividad investigadora en términos de desarrollo profesional (ofertas de empleo, pasantías, carrera profesional) puede constituir un aliciente importante para el fomento de la misma (18).

Aunque el tiempo se plantea siempre como primer argumento para justificar el no implicarse en tareas investigadoras, quizá el motivo básico sea realmente la falta de motivación $(86.3 \%$, ya que, si existe ésta, generalmente otros obstáculos pasan a segundo plano. Probablemente esto merecería un estudio que permitiera indagar en las motivaciones reales que llevarían a los profesionales de APS de Ica, a realizar investigación y sobre la presión asistencial medida en número de pacientes por día.

En nuestro estudio, además de los obstáculos enumerados líneas arriba se mencionan los de carácter organizativo o estructural; éstas junto con el escaso valor que le conceden a la formación en metodología de investigación podrían ser las causantes de que los profesionales en APS de Ica, se sientan en desventaja y limitados para investigar (barreras subjetivas). Básicamente, estos factores son los que suelen repetirse en otros estudios realizados con diversas poblaciones y con metodologías distintas $(7,16,17)$. A pesar de esto, la mayoría de los profesionales desearían desarrollar proyectos de investigación $(80.6 \%$ en el futuro $(19,20)$, principalmente porque consideran que la investigación $(95.3 \%$ es importante porque no es una pérdida de tiempo $(20,21)$. Esto refuerza la idea de que se hace obligatorio diseñar y desarrollar estrategias encaminadas a fomentar una cultura entre los profesionales que favorezca la idea de que la investigación es una tarea imprescindible e indiso- luble de los profesionales en APS (22). La reciente creación del Área de investigación en Atención Primaria de Salud de Ica (AICAP) ,como estructura de apoyo y la progresiva inclusión en ella de investigadores, con tiempos de dedicación específicas para la investigación, pueden contribuir a mejorar cualitativa y cuantitativamente la situación. $(18,23)$. Aun así, la medida de incentivación que fundamentalmente podría constituir un cambio decisivo sería el reconocimiento del papel de las actividades investigadoras en nuestra profesión por parte de las administración sanitaria (23) y su inclusión como un elemento más que considerar dentro de la carrera profesional (24). Los efectos de este paso necesario no sólo estarían encaminados a mejorar la autorrealización profesional y evitar el desgaste o anquilosamiento (25), sino sobre todo a mejorar la calidad y eficiencia de nuestra asistencia sanitaria, que en definitiva es lo que se trata. Nos ha sorprendido también de nuestro estudio el elevado porcentaje de profesionales de APS de Ica, que desconocen la existencia del AICAP como estructura de apoyo (83.1\% $/$ y de la tutorización de la investigación $(82.5 \%$; lo cual se explica porque esta iniciativa es reciente y aun no se han realizado reuniones de socialización, solo una comunicación oficial a las jefaturas de las Microredes de salud de la R.D N²10-2011-RED I.P.N/RR.HH, que resuelve su creación.

El pobre desarrollo de la investigación en APS de Ica, podría explicarse por el elevado número de barreras y dificultades que existen, que acabarían minando la actitud hacia la investigación.

El hecho de que los profesionales, tengan una actitud tan favorable hacia la investigación nos abre la puerta a la esperanza de un futuro mejor. Se debe de evitar que su actitud decline, con el paso del tiempo, adoptando estrategias que permitan la sostenibilidad de estas buenas actitudes como fortaleza para el desarrollo de la investigación en APS de Ica.

\section{REFERENCIAS BIBLIOGRÁFICAS.}

1 Arroyo-Hernández CH, De la Cruz W, Miranda-Soberón UE. Dificultades para el desarrollo de la investigación en pre grado, en una universidad pública de provincia, Perú. Rev Perú Med Exp Salud Pública. 2008;25 (4):348-49.

2. Arroyo-Hernández $\mathrm{CH}$, Zukerán-Medina $\mathrm{B}$, Miranda-Soberón UE. Análisis bibliométrico de la producción científica biomédica en la Región de Ica, Perú. 1998-2010. Rev Med Panacea. 2011:122-8.

3. Ministerio de Salud del Perú. Categorías de establecimientos del sector salud. N.T № 021-MINSA/DGSP-2005. V. 01 p.20.

4. Pons J, Navarro MD, Albert X, Ausejo M, Bolíbar B, Díaz CA, et al. La investigación en atención primaria. Barcelona: Agencia d'Avaluació de Tecnología i Recerca Mediques. CatSalut. Departament de Salut. Generalitat de Catalunya. Diciembre de 2004.

5. Díaz C, Manrique L, Galán E, Moisés S. Conocimientos, actitudes y prácticas en investigación de los estudiantes de pregrado de Facultades de Medicina del Perú. Acta Méd Per, enero-marzo, año/vol. 25, número 001 Colegio Médico del Perú Miraflores, Peru2008. pp. 9-15

6. Gavilán E, Pérula L, Jiménez L. Actitud hacia la investigación de residentes y tutores de medicina de familia en una unidad docente. Med fam Andal. 2006; 1: 10-16

7. Cevallos C, Garrido S, López MA, Cervera E, Estirado A. Investigación en atención primaria: actitud y dificultad percibidas por nuestros médicos. Aten Primaria. 2004; 34:520-7.

8. Aceituno H, Lanz JR, Lanz JA, Lander G. Interés, conocimientos, recursos y limitaciones de los estudiantes de medicina en la investigación científica. Gac Med Caracas. 1998; 106(1):40-46.

9. Hernández A, Morales O, Law Harrison L. Perspectivas sobre la investigación en enfermería en Guatemala. Enfermería glob.2006;8:1-10

10. Ramos $\mathbf{M}$, Sotomayor R. Realizar o no una tesis: razones de estudiantes de medicina de una universidad pública y factores asociados. Rev Per Med Exp Salud Pública. 2008; 25(3), 322-324.

11 Delgado R, Moreno L, González V. Conocimientos, actitudes y barreras hacia la investigación, las revisiones sistemáticas de la literatura y la colaboración Cochrane. Rev Colomb Anestesiol. vol. 34, núm. 4, 2006, pp. 227-231

12. Uribe J, Márquez C, Amador G, Chávez A. Percepción de la investigación científica e intención de elaborar tesis en estudiantes de psicología y enfermería. Enseñanza e investigación en psicología. 2011;16(1): 15-26

13. Díaz G, Palmeiro G, Portuburo M, Vásquez L, Rúas A. Factores que influyen en la producción científica en atención primaria. Cad Aten Prim. 2007; 13(1): 169-172.

14. Llovera J. Reflexionando sobre los obstáculos en la investigación de atención primaria. Opinión de los investigadores. En: Documentos de trabajo sobre investigación en Atención Primaria. Madrid: FIS, 1996.

15. Jowett SM, Macleod J, Wilson S, Hobbs FD. Research in primary care: extent of involvement and perceived determinants among practitioners from one English region. $\mathrm{Br}$ J Gen Pract 2000; 50: 387-9.

16. Rosemann T, Szecsenyi J. General practitioners' attitudes towards research in primary care: qualitative results of a cross sectional study. BM C Fam Pract.

2004; 5:31

17. Fernández de Sanmamed MJ, De la Fuente JA, Mercader J, Borrell C, Martín C, et al. Factores relacionados con la actividad investigadora de los médicos de atención primaria. Aten Primaria 1997; 19: 283-9. 
18. Fernández Fernández I. ¿lnvestigación en atención primaria? Editorial semFYC. Aten. prim. (Barc., Internet) 2003; 31: 281-4.

19. Torres $\mathbf{M}$, Jódar Hoste $E$, Jiménez Gómez $\mathrm{N}$, Casagran A. Investigación biomédica en atención: la perspectiva de los profesionales. Aten. prim. (Barc., Internet) 2004; 34 (Extraordin 1): 131

20. Robinson G. What are the attitudes of general practitioners toward research? Brit J Gen Pract. 2000; 50: 390-2.

21 Askew DA, Clavarino AM, Glasziou PP, Del Mar CB. General practice research: attitudes and involvement of Queensland general practitioners. Med J Aust. 2002; 177: 74-7.

22. Jiménez J. Necesitamos más y mejor investigación en atención primaria. Aten Prim. 2004; 34: 520-7.

23. Gavilán E. El residente como investigador: dificultades, condicionantes y posibles soluciones. Tribuna Docente 2003; 4: 68-71

24. Extremera F, Gómez Gascón T, Martín Zurro A, Monset L, Soler M, Veras R, et al. La carrera profesional en atención primaria. Barcelona: semFYC: 8.

25. Melguizo $M$, Prados MA, Esquisabel R, Bueno JM, Kloppe P, López García-Franco A, et al. Medidas de motivación e incentivación en atención primaria. Barcelona: semFYC. 1993; 1
FINANCIAMIENTO: Autofinanciado.

AGRADECIMIENTOS: Al equipo multidisciplinario de profesionales en atención primaria de los diferentes establecimientos que conforman la Red de Salud de Ica.

CONTRIBUCIONES DE AUTORíA: BBC participo en el diseño del estudio, el análisis de los datos, revision crítica del artículo y aprobación de la versión final.

\section{Attitudes and barriers on research in primary health care of Ica}

O

bjective: Knowing the attitudes and barriers on research, which were perceived by the professionals in APS of Ica. Materials and methods: A Descriptive Study-relational and cross-sectional, the sample was represented by 191 professionals, who were selected at random, 53 establishments in the first level of care . To establish quantitatively the level of attitudes and perception of barriers was applied a Likert-type scale questionnaire respectively. Results: It was found a positive attitude in $95.8 \%$ of the total sample, The best attitudes were: to investigate is not necessary to be "a scientist" $(87.5 \%)$, wish to expand in the future their knowledge in MIC (917\%Would time out of your workday, to investigate $(85.8 \%)$, they are interested in research $(89.5 \%$ etc. The $96.2 \%$ of the total respondents perceive in high level, barriers to implementation of the research; The obstacles identified were lack of time $(617 \%)$, lack of motivation (86.3\%), there are no support structures (83.1\%), etc. Conclusions: In APS of Ica, there are favorable attitudes toward research and perceived high levels of barriers to implementation.

Key words: Primary Health Care, Research, attitudes, barriers. (source: MeSH NLM)

CITA.

Becerra-Canales B. Actitudes y barreras sobre investigación en atención primaria de salud de Ica. Rev méd panacea.2013; 3 (1):6-10. 